\title{
Is Ride-sourcing Service really Sustainable : Operational effi- ciency and Social Welfare Analysis through an equilibrium model
}

\author{
Zipeng Zhang * and Ning Zhang
}

School of Economics and Management, Beihang university, Beijing 100191, PR China;

\begin{abstract}
With increasing availability of alternative mobility options for city transportation system, it is necessary to better understand how emerging mobility are impacting the travel demand and consumer-social surplus. However, few study has been conducted to evaluate the social welfare effects of the range of vacant trip in mobility oriented development(MOD) mode. This paper identified the vacant trip and loading rate evaluation model under mobility oriented development mode. recommended solutions to enhance the effective operation of the different mobility services under numerical illustrations, which can offer some beneficial guidance and theoretic basis to the efficiency of MOD in planning and management aspects.
\end{abstract}

Keywords: Vacant trip; Cruising Taxis service; ride sourcing service

\section{Introduction}

Mobility oriented development (MOD) has grown tremendously in recent years as a renewed interest in urbanism and growing environmental pollution, terrible congestion, and economic concerns (Ma et al. 2020). Simultaneously, dynamic ride-sourcing service(RSS) (e.g. Didi Chuxing in China, Uber and Lyft in U.S.) have emerged due to the advances in mobile and GPS technologies, which have made traffic mobility easier and more efficient(Wang, Ban, and Huang 2019; Xu and Meng 2019). Meanwhile, some incentive public policies, the priority mechanism for high-occupancy vehicle/toll (HOV/HOT) lanes, Price compensation and security enhancement, are being used to encourage commuters to change from driving alone to ridesharing and public transit mode for easing the negative impact of traffic congestion and enhancing mobility.

These RSS platform proclaim that they aims to transfer the inefficient cruise-street hailing mode to convenient matching-waiting mode by breaking the asymmetric supplydemand information in mono-poly of the traditional cruising taxis service(CTS), and then achieve the objective of decreasing empty trip and pollution emissions. But the continuous competition in mobility market produces many bad results, the recent evidence shows that the expansion of RSS markets has already contributed substantially to the deteriorating traffic conditions in some major cities because of drivers' profitable selfishness(Erhardt et al. 2019), which generate massive vacant trips while providing additional service than road capacity during peak hours in morning and evening commute. Furthermore, RSS also brings some negative impact on the CTS market: decrease in income of CTS drivers, losses of CTS staff increasing protest activities, contradictions between customers and drivers in price. It Is the top priority issue to balance the system among TTS, RSS and passenger for the government.

RSS and CTS studies can be broadly classified into two types:one, modeling equilibrium of trip-based demand assignment considering different properties between RSS and TTS, which is often formulated as an network optimization problem; and the orther one, 
optimization of endogenous factors influencing Equili-brium network (e.g.,vacant trips; waiting time; dynamic pricing stragegy; service, safety).

The trip-base travel demand Equilibrated mechanism in TTS system has been made to better understand and characterize with the continuous efforts by researchers(Wong, Wong and Yang 2001; Yang et al. 2005), unlike the conven-tional four-step process for demand forecast above, recently, some big date methods are used to estimate the dynamic RSS supply-demand condition with multiple data sources (e.g.,history RSS orders, weather and real-time traffic data) to achieve a better system utilities (Zhou et al. 2019; Wang et al. 2017).

Besides, in the research of endogenous factors Optimization, (Sun et al. 2019) and (Wang et al. 2020) focused on the design of prices and subsidies on two-sides RSS/TTS market, And suggested that RSS platforms should carry out a strong incentive to encourage sharing via subsidies. Meanwhile some researcher introduce a price competition model between RRS and TTS market, and found that a win-win outcome for them can be achieved by a dynamic payout ratio (Ramezani and Nourinejad 2018).

In this paper, we discusses various urban modes of transport (e.g. automobile, mass transit) in the context of emerging technical (app-based RSS, autonomous vehicles) and organizational (e-reserved-based ridesharing service (ERS) mode, autonomous car-sharing service (ACS) mode, customer centric mobility) solutions for the mobility as a service(MaaS). we presents vacant trip related social welfare analysis model to determin the system optimal for a better transportation-related city management proposing Mobility Oriented Development.

The rest of the paper is organized as follows. Section 2 presents the mobility network structure and the vacant trips formulations for three different mobility mode. In section 3 , the social welfare and loading rate in CTS, ACS, ERS system are discussed, respectively. Section 4 examines and analyses the performance of mobility system under different loading rate, numerical illustrations and verifications are also presented in this section. Finally we give the conclusion.

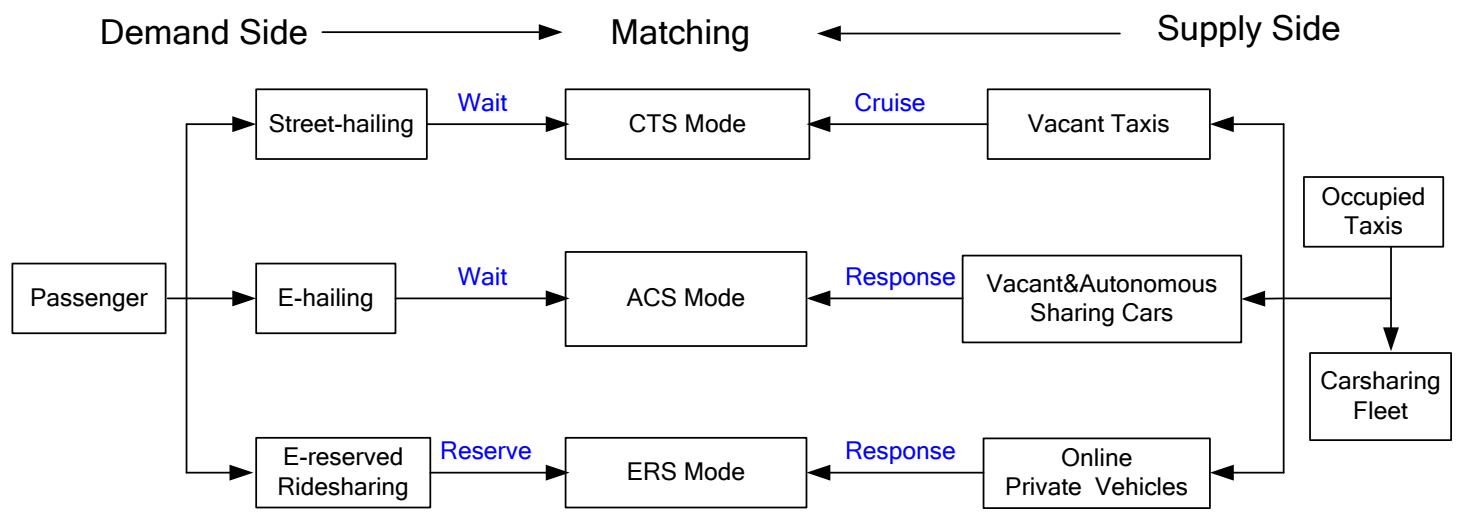

Figure 1 The competitive traffic mobility market between traditional taxi service and ride-sourcing service texts

\section{Modeling Structure}

As mentioned before, the major in the MOD system are passenger-vehicle matching and occupied/empty vehicle movements over urban road network. Thus, the analyses should involve not only the mobility system itself but also the urban road network.

\subsection{Mobility Network Presentation}

Supposing $k(k=3)$ types of mobility service, traditional cruising taxis service (CTS), emerging autonomous car-sharing service (ACS) and e-reserved based ridesharing/ carpooling service (ERS), driving in a road network $G(V, A)$, where $V$ is the set of nodes and $A$ is the set of links in the network. Corresponding to this, there are also three groups of ride-sourcing customer demands exist in the network: street-hailing passengers, 
e-hailing passengers, e-reserved rider-sharing passengers. The passenger-vehicle matching mode among different service are shown in Fig. 1. In any given time, the number of customers demanding ride from origin zone $i$ to destination zone $j$ is $q_{i j}$. In this paper, $q_{i j}$ is assumed to be a function of customer-waiting time at origin zone $i$, the monetary price and travel time cost for traffic from zone $i$ to zone $j$, Moreover, the directed links are weighted with routing probabilities for different trip mode, $p_{i j}^{k}$, to describe the routing choices by vehicles, let $u_{i j}$ as the optimal travel path between zoon $i$ and zone $j$ , which yields $u_{i j}=\min \left(p_{i j}, p_{i j}, \in A\right)$. Donate $I$ and $J$ as the sets of customer origin and destination zones, respectively, which yields $I, J \subset V$, then we have the following trip end constraints which following the Gumbel distribution when achieving system equilibrium.

$$
\begin{array}{ll}
O_{i}=\sum_{j \in J}^{k} q_{i j}^{k}, & i \in I \subset V, k=1,2,3 \\
D_{j}=\sum_{i \in I}^{k} q_{i j}^{k}, & j \in J \subset V, k=1,2,3
\end{array}
$$

where $O_{i}$ and $D_{j}$, are respectively the total traffic supply in origin zone $i \in I$ and demand for destination zone $j \in J$, meanwhile, $k$ is the different group of traffic mobility, $q_{i j}^{1} q_{i j}^{1} q_{i j}^{3}$ indicate the different mobility types of demand for the CTS, ACS and ERS from origin zone $i \in I$ to destination zone $j \in J$. Obviously, the sum of $q_{i j}^{1}, q_{i j}^{1}$ and $q_{i j}^{3}$ equal $D_{i j}$.

\subsection{Mobility Demand Description}

Passenger demand for mobility trips is assumed to be related to the trip price, and to a proxy for service quality. The aspect of service quality of particular interest in this paper is the expected delay/waiting time incurred in engaging a mobility mode we can get the demand increased prices and waiting times will inhibit passenger demand, according to the research(Wong, Wong and Yang 2001; Djavadian and Chow 2017), the waiting time is determined by the total vacant mobility-hours available, the more empty mileage will lead to the greater of the probability that the vacant taxi move on the road, it will be easier for passenger-taxi matching. Assuming the total customers' demand of trip mileage, $D$, to be a strictly decreasing and convex function of the trip fare $p_{i j}^{k}$ and passengers' waiting time $t_{i j}^{k}$, then we can have the Cobe-Douglas production expression form(Douglas 1972) of the demand function as below:

$$
\begin{aligned}
& D_{i j}=\sum_{i, j \in V}^{k} q_{i j}^{k}=\sum_{i, j \in V}^{k} \omega\left(f_{i j}^{k}\right)^{\alpha}\left(t_{i j}^{k}\right)^{\beta}, \quad k=1,2,3 \\
& t_{i j}^{k}=\xi\left(v_{i j}^{k}\right)^{\gamma} \quad i, j \in V, k=1,2,3
\end{aligned}
$$

Where $t_{i j}^{k}$ is the waiting time for different groups of passenger who have the demand from origin $i \in I$ to destination $j \in J$. In this paper, it will be the street-hailing, ehailing and e-reserved ridesharing waiting time in CTS, ACS and ETS mode when $k=1$ ,$k=2$ and $k=3$, respectively. Furthermore, $v_{i j}^{k}$ is the empty mileage of different mobility service mode in the same OD matching pair, and let $\alpha, \beta, \gamma$ be defined as the elasticity of price, waiting time and empty mileage respectively, according to the Cobe-Douglas production function and the realistic knowledge, we can conclude that the $\alpha, \beta, \gamma$ yields $\alpha<0, \beta<0, \gamma<0 . \omega, \xi$ represent the demand elasticity coefficient, which can be determined by the economic and consumer condition, spatial layout, road network characteristics and other factors of the city. Combing Eq.(4) and (3), we can get the Cobe-Douglas production demand function in car-based mobility market:

$$
D_{i j}=\sum_{i, j \in V}^{k} \omega \xi^{\beta}\left(f_{i j}^{k}\right)^{\alpha}\left(v_{i j}^{k}\right)^{\beta \gamma}, \quad i, j \in V, k=1,2,3
$$


We also consider a realistic mobility fare structure consisting of three components: a flag-fall or a constant initial flat charge, a distance-based charge, and a delay-based charge. Let $\left(\tau^{k}, p_{m}, \sigma^{k}, \rho^{k}\right)$ be the initial flag-fall charge per ride, maximum mileage for flag-fall charge, fare charge per occupied unit mileage and fare charge per congestion delay time for different matching mode, respectively. And donate $t_{i j} t_{i j}^{*}$ as the ride time from region $i$ to region $j$. We have congestion delay $T_{i j}=t_{i j}-t_{i j}^{*}$ Then the mobility fare in single trip $p_{i j}$ for different mode can be described as below:

$$
f_{i j}^{k}=\tau^{k}+\sigma^{k} \cdot p_{i j}^{k}+\rho^{k} \cdot T_{i j}, \quad k=1,2,3
$$

The different fare for three mode of matching can be established into varied combination to satisfy different demand condition. For the CTS trips, we have the charging function:

$$
f_{i j}^{1}= \begin{cases}\tau^{1} & \text { if } p_{i j}<p_{m} \\ \tau^{1}+\sigma^{1} \cdot\left(p_{i j}-p_{m}\right)+\rho^{1} \cdot\left(t_{i j}-t_{i j}^{*}\right), & \text { if } p_{i j}>p_{m}\end{cases}
$$

For the ACS trips, we have the charging function:

$$
f_{i j}^{2}= \begin{cases}\tau^{1} & \text { if } \sigma^{2} \cdot p_{i j}+\rho^{2} \cdot t_{i j}<\tilde{p} \\ \sigma^{2} \cdot p_{i j}+\rho^{2} \cdot t_{i j}, & \text { if } \sigma^{2} \cdot p_{i j}+\rho^{2} \cdot t_{i j}>\tilde{p}\end{cases}
$$

Where $\tilde{p}$ is the minimum fare charge for single trip. Meanwhile, Donate $\lambda$ as the service frequency/ride-sharing number during ridesharing drivers' whole own trip, and let $\theta$ as the coefficient of ride-sharing number for initial flag-fall charge per ride. Then we have the charging function of ERS trips:

$$
\begin{aligned}
& f_{i j}^{3}= \begin{cases}\theta \tau^{3} & \text { if } p_{i j}<p_{m} \\
\theta \tau^{3}+\sigma^{3} \cdot\left(p_{i j}-p_{m}\right) & \text { if } p_{i j}>p_{m}\end{cases} \\
& \theta= \begin{cases}1 & \text { if } \lambda=1,2 \\
1.5 & \text { if } \lambda \geq 3\end{cases}
\end{aligned}
$$

\subsection{Mobility Vacant Trips Analysis}

In this section, we will discuss the difference of vacant trips among CTS, ACS and ERS.The vacant trips develops because of the spatial distance between the next pickup point and last dropoff point. The cruising taxi drivers search and match passenger by the experienced characteristics of demand distribution, the by contrast, ride-sourcing drivers can select the optimal matching result to minimize their empty miles (maximum their trip utilities ) by obtaining the OD information of potential passengers by advance. The e-reserved ridesharing mode will be follow the dispatch of platform, it is more effective to attract more potential private vehicles and passsengers to using ride-sourcing service by matching the similar OD route.

\subsubsection{CTS Vacant Trips}

The destination choice of CTS mode mainly depends on the choice behavior of taxi drivers. It is assumed here that, taxi drivers are completely rational, they always make a choice according to the principle of maximum passengers matching probability with the minimum vacant mileages., there are a maximum of four target search zones for CTS driver, once the last ride is completed, who is assumed to attempt to minimize individual expected vacant time/distance required to meet the next customer. The CTS drivers will meet two choice strategies seeking the next customer: empiricism cruising and random cruising. For the first one, CTS drivers prefer cruising the zone where there are maximum traffic demand among other three regions. For the second mode, the expected search time in each zone is a random variable due to variations in perceptions of taxi drivers and the random arrival of customers. This random variable is assumed to be identically distributed with a Gumbel density function. With these behavior assumptions, the probability that a vacant taxi originating at zone $j \in J$ chooses to meet a customer at zone $i \in I$ is specified by the following logit model: 


$$
P_{j i}=\frac{\exp \left\{-\kappa\left(u_{j i}-\chi O_{i}\right)\right\}}{\sum_{m, i \in I, j \in J} \exp \left\{-\kappa\left(u_{j m}-\chi O_{m}\right)\right\}}
$$

when $i=j$ represents the probability of a cruising taxi that takes a customer to zone $i$ to search and meet the next customer in the same zone, and $\kappa$ is a nonnegative parameter which can reflect the degree of uncertainty on customer demand and mobility services. The probability is in turn affected by the customer demand Eq.(5), therefore, it can also reflect a driver's trade-off between customer demand and proximity. $\chi$ is the conversion coefficient from the traffic demand to traffic mileage. $v_{i i}$ is the

The total expectation empty mileage for vacant taxi cruising from the zone $j \in J$ to the zone $i \in I$

$$
u_{j}=\sum_{i \in I, j \in J} u_{j i} P_{j i}
$$

According to the Eq.(2), the occupied taxis will change to the vacant one, the new vacant vehicle in zone $j \in J$ after one OD trip can be express:

$$
E_{j}=D_{j}=\sum_{i \in I} q_{i j}
$$

Combining Eq.(11),(12),(13), we can get the cruising-based vacant mileage, $v_{i j}^{1}$ :

$$
v_{i j}^{1}=\sum_{j \in J} E_{j} u_{j}=\sum_{j \in J} \sum_{i \in I} q_{i j} \sum_{i \in l} u_{j i} P_{j i}
$$

\subsubsection{ACS Vacant Trips}

We assume the ACS mode can only carry one passenger in each trip service, the vacant sharing cars usually choose the shortest path to reach the pickup zone when they respond to the trip demand by fully communicating with the passengers in advance with the smart phone app, and the empty mileage will not effect by the uncertain cruise behavior. At the same time, the automonous car-sharing service mode can master the damand's OD information and the congestion condition of road network through the ridesourcing service to help them ignore the impact of uncertainties(the uncertainties parameter $\kappa$ can not be taken into account). Referring to Eq. (11), it can be obtained that the probability and the expectation empty mileage for ACS originating at zone $j \in J$ chooses to meet a customer at zone $i \in I$ :

$$
\begin{aligned}
& P_{j i}^{*}=\frac{\exp \left(-u_{j i}\right)}{\sum_{m \in I} \exp \left(-u_{j m}\right)} \\
& u_{j}^{*}=\sum_{i \in I} u_{j i} P_{j i}^{*}, \quad i \in I, j \in J
\end{aligned}
$$

Combining Eq.(1),(15),(16),we can get the vacant mileage of ACS

$$
v_{i j}^{2}=\sum_{j \in J} E_{j} u_{j}^{*}=\sum_{j \in J} \sum_{i \in \backslash \backslash j} q_{i j} \sum_{i \in \backslash \backslash j} u_{j i} P_{j i}^{*}
$$

\subsubsection{ERS Vacant Trips}

The ERS mode can be regard as one of the shared mobility, the matching willingness of ERS will become stronger during the rush hour of long-distance commute. Unlike CTS or ACS mode, driver can provide three or four empty seats even more frequency of getting on and off ridesharing occupancy during their own trips in this mode. The maximun utilities for ridesharing driver is more service frequency under the less devistion from his own original shortest route. We know $\lambda$ can express the service frequency/ridesharing number during ridesharing trip in ERS. So the total expectation empty mileage for vacant private car cruising from the zone $j \in J$ to the zone $i \in I$ in ERS mode can be expressed as:

$$
\begin{aligned}
& u_{j}^{* *}=\sum_{i \in I}^{\lambda} u_{j i}^{\lambda} P_{j i}^{*}, \quad j \in J, \lambda \geq 1 \\
& v_{i j}^{3}=\sum_{j \in J} E_{j} u_{j}^{* *}=\sum_{j \in J} \sum_{i \in V \backslash j}^{\lambda} q_{i j} \sum_{i \in V \backslash j}^{\lambda} u_{j i}^{\lambda} P_{j i}^{*}
\end{aligned}
$$




\section{Social welfare and loading rate analysis}

In this section, we introduce a aggregate producer-consumer surplus theory of Douglas production(Douglas 1972) model to analyze and improve the performance of the mobility market.

\subsection{Social welfare analysis model}

According to the theory above, the production surplus, $S^{p}$, is equal to the difference between total revenue and total cost, we can obtain the total mobility production surplus function for three mobility service:

$$
S^{p}=\sum_{i, j \in V}^{k} S_{i j}^{k}=\sum_{i, j \in V}^{k} f_{i j}^{k} \cdot q_{i j}^{k}-\sum_{k=1,2,3} C^{k}
$$

Where $S_{i j}^{k}, k=1,2,3$ represents the production sur-plus of CTS, ACS and ETS, respectively. And $C^{k}$ is the operation cost function for different mobility service mode, which satisfied the condition as below:

$$
C^{k}=\sum_{i, j \in V}^{k} c^{k}\left(q_{i j}^{k}+v_{i j}^{k}\right) \quad k=1,2,3
$$

$c^{k}$ is the unit energy cost of trip mileages, and The unit energy cost is different among different mobility service, for example, the source of energy is natural gas in traditional CTS mode, while electric vehicles are mainly engaged in ACS mode and drives are with the pressure of high-gasoline price in ETS network. so $c^{k}$ yields $c^{2}<c^{1}<c^{3}$.

According to pasenger demand function(5), we can get the realtionship between the fare price and travel demand as shown in Eq.(18), and the total trip fare, $p$, can be denote as below:

$$
p=\sum_{i, j \in V}^{k} f_{i j}^{k}=\left(\sum_{i, j \in V}^{k} \frac{D_{i j}}{\omega \xi^{\beta}\left(v_{i j}^{k}\right)^{\beta \gamma}}\right)^{\frac{1}{\alpha}}, \quad k=1,2,3
$$

then the passengers' consumer surplus function (PCS) is:

$$
S^{c}=\int_{0}^{D}\left(\sum_{i, j \in V}^{k} \frac{x}{\omega \xi^{\beta}\left(v_{i j}^{k}\right)^{\beta \gamma}}\right)^{\frac{1}{\alpha}} d x-p D_{i j}=\left\{\begin{array}{cc}
\left(\sum_{i, j \in V}^{k} \frac{1}{\omega \xi^{\beta}\left(v_{i j}^{k}\right)^{\beta \gamma}}\right)^{\frac{1}{\alpha}}\left(\left.\frac{x^{\frac{1}{\alpha}+1}}{\frac{1}{\alpha}+1}\right|_{0} ^{D}\right)-p D_{i j}, \quad \alpha \neq-1 \\
\left(\sum_{i, j \in V}^{k} \frac{1}{\omega \xi^{\beta}\left(v_{i j}^{k}\right)^{\beta \gamma}}\right)^{\frac{1}{\alpha}}\left(\left.\ln x\right|_{0} ^{D}\right)-p D_{i j}, \quad \alpha=-1 \\
\left(\sum_{i, j \in V}^{k} \frac{1}{\omega \xi^{\beta}\left(v_{i j}^{k}\right)^{\beta \gamma}}\right)^{\frac{1}{\alpha}}\left(\frac{D^{\frac{1}{\alpha}+1}}{\frac{1}{\alpha}+1}\right)_{\infty,}-p D_{i j}, \quad \alpha<-1 \\
-1 \leq \alpha<0
\end{array}\right.
$$

According to the research(Wong, Wong, and Yang 2001), although we can observe the occurrence in some situation for price elasticity coefficient of $\alpha \geq-1$, but it will be more close to the reality when the price elasticity meet the condition of $\alpha<-1$.

This paper denies social welfare of the mobility service market as the summation of net benefits as given by the aggregate producer-consumer surplus:

$$
S=p D_{i j}-\sum_{k=1,2,3} C^{k}+\left(\sum_{i, j \in V}^{k} \frac{1}{\omega \xi^{\beta}\left(v_{i j}^{k}\right)^{\beta \gamma}}\right)^{\frac{1}{\alpha}}\left(\frac{D^{\frac{1}{\alpha}+1}}{\frac{1}{\alpha}+1}\right)-p D_{i j}=\left(\sum_{i, j \in V}^{k} \frac{1}{\omega \xi^{\beta}\left(v_{i j}^{k}\right)^{\beta \gamma}}\right)^{\frac{1}{\alpha}}\left(\frac{D_{i j}^{\frac{1}{\alpha}}}{\frac{1}{\alpha}+1}\right)-\sum_{i, j \in V}^{k} c^{k}\left(q_{i j}^{k}+v_{i j}^{k}\right)
$$


From Eq.(24), it can be seen that the social welfare of the mobility market has nothing to do with the payment fee, which means that the passenger payment fee has been transferred in the mobility system, and the variable of social welfare function is only the vacant trip and passenger demand.

\subsection{Loading rate analysis model}

The optimal loading rate(OLA) of mobility service is the result of the comprehensive function of price, service level and other modes in the mobility market. A reasonable loading rate is related to the parameters of fare charging level, waiting time and vacant mileage, excessive loading rate will lead to long-time waiting for passengers, which easily result in a sharp decline for the mobility service level, while a lower one will affect the initiative of mobility service providers, causing the idle mobility resources. So it is necessary to explore the optimal loading rate of mobility market under the maximum social welfare condition in order to achieve the objective of sustainable development.

We can specify a point of constrained welfare maximisation for the system. This can be derived by maximising $S$, where

$$
\begin{aligned}
\frac{\partial S}{\partial D_{i j}} & =\left(\sum_{i, j \in V}^{k} \frac{D_{i j}}{\omega^{k}\left(\xi^{k}\right)^{\beta}\left(v_{i j}^{k}\right)^{\beta \gamma}}\right)^{\frac{1}{\alpha}}-c^{k}=0 \\
\frac{\partial S}{\partial v_{i j}^{k}} & =\left(\sum_{i, j \in V}^{k} \frac{1}{\omega^{k}\left(\xi^{k}\right)^{\beta}}\right)^{\frac{1}{\alpha}}\left(\frac{D^{\frac{1}{\alpha}+1}}{\frac{1}{\alpha}+1}\right)\left(-\frac{\beta \gamma}{\alpha}\right)\left(v_{i j}^{k}\right)^{\frac{\beta \gamma}{\alpha}-1}-c^{k}=0
\end{aligned}
$$

Combing Eq.(25),(26), we can calculate the optimal trip mileage, $D^{*}$, and optimal vacant mileage, $\left(v_{i j}^{k}\right) *$, with the maximum social welfare:

$$
\begin{aligned}
& \left(v_{i j}^{k}\right)^{*}=\left(-\frac{\omega \xi^{\beta} \beta \gamma\left(c^{k}\right)^{\alpha}}{1+\alpha}\right)^{\frac{1}{1-\beta \gamma}} \\
& D^{*}=\left(-\frac{1+\alpha}{\beta \gamma}\right)\left(-\frac{\omega \xi^{\beta} \beta \gamma-\left(c^{k}\right)^{\alpha}}{1+\alpha}\right)^{\frac{1}{1-\beta \gamma}}
\end{aligned}
$$

The loading rate can be adopt as below:

$$
R^{*}=\frac{D^{*}}{D^{*}+\left(v_{i j}^{k}\right)^{*}}
$$

\section{Numerical Analysis}

\subsection{Network and Key Parameters}

The date of network used in the analysis of this work is the well-known Sioux Falls network, which be seen in Fig. 2. This network contains 24 nodes (all of which are origins and destinations) and 76 links. Again the demand is assumed to be normally distributed, with a specified variance; demand is also truncated at zero to ensure non-negativity. In this paper we select 7 note as a small trips network show in Fig. 2 red box. The data in Table 3, Table 4 and Table 5 can be calculate combining to the Table 1 and Table 2

According to the research statistical data of Shenzhen in China, the number of issued vehicles in mobility mode of CTS and ACS is 62313 and 21489 respectively in 2019. For ACS mode, the daily average order of ECS is about 9.3, the daily average driving mileage and loading mileage is about $84.0 \mathrm{~km}, 73.2 \mathrm{~km}$ respectively. Mean-while, and the daily average order operation amount of each car is about $¥ 296.2$. By contrast, For CTS mode the daily average order of CTS is about 35.1, the daily loading mileage is about $384.5 \mathrm{~km}$, and the daily average order operation amount of each car is about ¥1027. Refer to some parameters definitions [5][13], the key parameters can be shown in Table 6. 


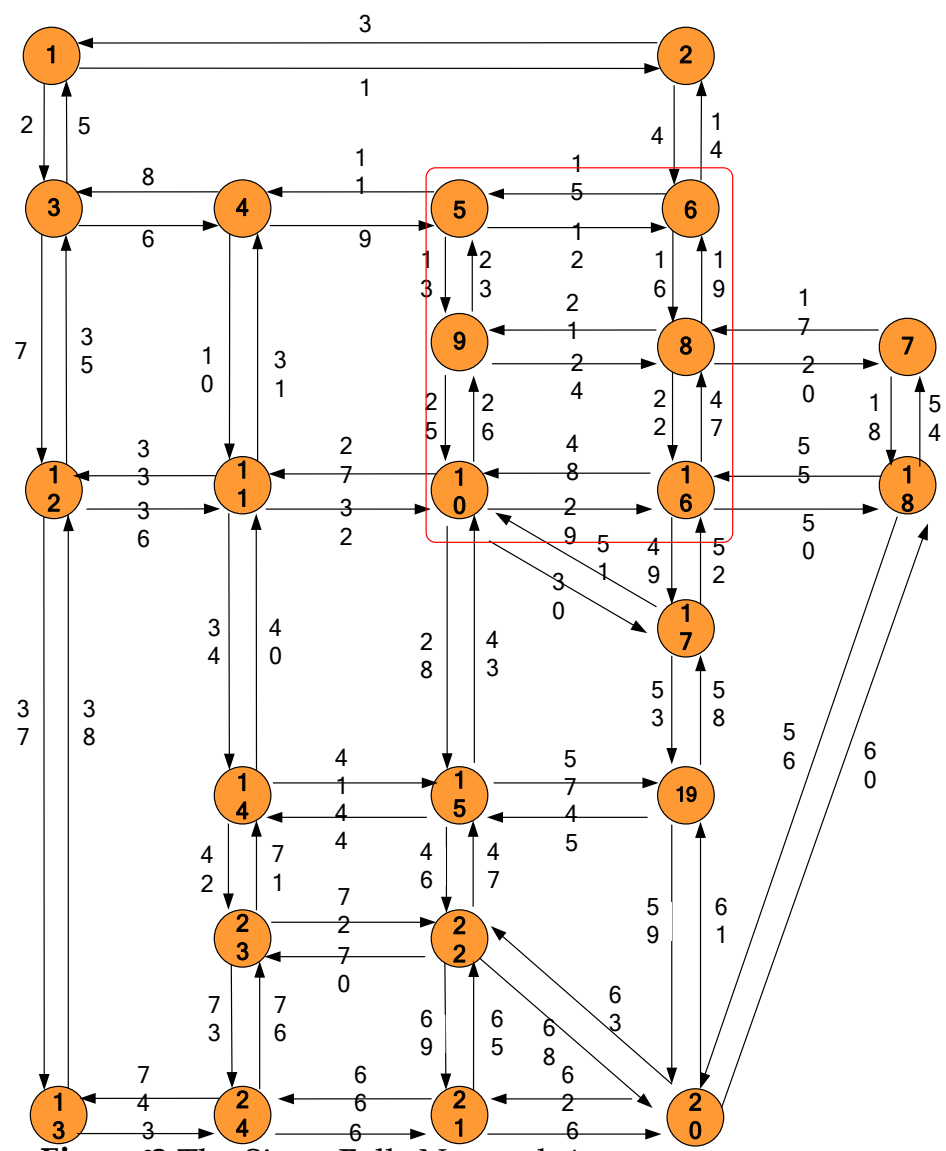

Figure92 The Sioux Falls Network 4

Table 1 The optimal travel distance in network

\begin{tabular}{ccccccc}
\hline Zone & $\mathbf{5}$ & $\mathbf{6}$ & $\mathbf{9}$ & $\mathbf{8}$ & $\mathbf{1 0}$ & $\mathbf{1 6}$ \\
\hline $\mathbf{5}$ & $\mathbf{0}$ & $\mathbf{1 9}$ & $\mathbf{2 5}$ & $\mathbf{3 3}$ & 27 & 31 \\
6 & 20 & 0 & 15 & 23 & 17 & 21 \\
9 & 28 & 16 & 0 & 17 & 24 & 28 \\
8 & 36 & 24 & 17 & 0 & 16 & 23 \\
10 & 33 & 18 & 24 & 17 & 0 & 0 \\
16 & 20 & 21 & 27 & 30 & 22 & 26 \\
\hline
\end{tabular}

Table 2 The optimal travel distance in network

\begin{tabular}{ccccccc}
\hline Zone & $\mathbf{5}$ & $\mathbf{6}$ & $\mathbf{9}$ & $\mathbf{8}$ & $\mathbf{1 0}$ & $\mathbf{1 6}$ \\
\hline $\mathbf{5}$ & $\mathbf{0}$ & $\mathbf{3 0}$ & $\mathbf{3 5}$ & $\mathbf{2 5}$ & $\mathbf{2 0}$ & $\mathbf{3 0}$ \\
$\mathbf{6}$ & $\mathbf{2 0}$ & $\mathbf{0}$ & $\mathbf{1 5}$ & $\mathbf{2 0}$ & $\mathbf{1 5}$ & $\mathbf{4 0}$ \\
9 & $\mathbf{4 0}$ & $\mathbf{3 0}$ & $\mathbf{0}$ & $\mathbf{2 5}$ & $\mathbf{4 0}$ & $\mathbf{2 0}$ \\
$\mathbf{8}$ & $\mathbf{3 0}$ & 35 & 25 & 0 & 35 & 15 \\
10 & 20 & 15 & 20 & 30 & 0 & $\mathbf{2 0}$ \\
16 & 30 & 25 & 30 & $\mathbf{2 0}$ & $\mathbf{2 0}$ & $\mathbf{0}$ \\
\hline
\end{tabular}

Table 3 The probability of different search directions in CTS

\begin{tabular}{ccccccc}
\hline Zone & 5 & $\mathbf{6}$ & $\mathbf{9}$ & $\mathbf{8}$ & $\mathbf{1 0}$ & $\mathbf{1 6}$ \\
\hline $\mathbf{5}$ & $\mathbf{0}$ & $\mathbf{0 . 4 8 0}$ & $\mathbf{0 . 0 8 1}$ & $\mathbf{0 . 0 0 7}$ & $\mathbf{0 . 0 4 3}$ & $\mathbf{0 . 0 2 4}$ \\
$\mathbf{6}$ & $\mathbf{0 . 1 0 4}$ & $\mathbf{0}$ & $\mathbf{0 . 4 6 8}$ & $\mathbf{0 . 0 4 2}$ & $\mathbf{0 . 2 4 5}$ & $\mathbf{0 . 1 3 7}$ \\
9 & $\mathbf{0 . 0 2 0}$ & $\mathbf{0 . 5 1 7}$ & $\mathbf{0}$ & $\mathbf{0 . 3 8 9}$ & $\mathbf{0 . 0 4 8}$ & $\mathbf{0 . 0 2 6}$ \\
$\mathbf{8}$ & $\mathbf{0 . 0 0 2}$ & $\mathbf{0 . 0 4 8}$ & $\mathbf{0 . 4 0 1}$ & $\mathbf{0}$ & $\mathbf{0 . 5 3 4}$ & $\mathbf{0 . 0 1 5}$ \\
10 & $\mathbf{0 . 0 2 1}$ & $\mathbf{0 . 3 4 1}$ & $\mathbf{0 . 0 5 6}$ & $\mathbf{0 . 4 4 7}$ & $\mathbf{0}$ & $\mathbf{0 . 1 3 3}$ \\
16 & $\mathbf{0 . 0 1 5}$ & $\mathbf{0 . 4 1 1}$ & $\mathbf{0 . 0 6 9}$ & $\mathbf{0 . 0 2 8}$ & $\mathbf{0 . 3 0 4}$ & $\mathbf{0}$ \\
\hline
\end{tabular}

Table 4 The probability of different search directions in ACS

\begin{tabular}{ccccccc}
\hline Zone & 5 & 6 & 9 & 8 & 10 & 16 \\
\hline 5 & 0 & 0.730 & 0.002 & 0.000 & 0.000 & 0.000 \\
6 & 0.006 & 0 & 0.862 & 0.000 & 0.117 & 0.016 \\
9 & 0.000 & 0.731 & 0 & 0.269 & 0.000 & 0.000 \\
8 & 0.000 & 0.000 & 0.269 & 0 & 0.731 & 0.000 \\
10 & 0.000 & 0.265 & 0.001 & 0.721 & 0 & 0.013
\end{tabular}




\begin{tabular}{lllllll}
16 & 0.000 & 0.704 & 0.002 & 0.000 & 0.259 & 0 \\
\hline
\end{tabular}

Table 5 The probability of different search directions ERS

\begin{tabular}{ccccccc}
\hline Zone & 5 & 6 & 9 & 8 & 10 & 16 \\
\hline 5 & 0 & 0.890 & 0.000 & 0.000 & 0.000 & 0.000 \\
6 & 0.006 & 0 & 0.572 & 0.000 & 0.117 & 0.000 \\
9 & 0.000 & 0.700 & 0 & 0.269 & 0.000 & 0.000 \\
8 & 0.000 & 0.000 & 0.269 & 0 & 0.031 & 0.000 \\
10 & 0.000 & 0.265 & 0.001 & 0.721 & 0 & 0.013 \\
16 & 0.000 & 0.704 & 0.002 & 0.000 & 0.059 & 0 \\
\hline
\end{tabular}

As analysis model in section 3 above, the app-based mobility mode such as ACS and ECS shortens the vacant driving time, improves the loading rate and prolongs the waiting time of passengers. However, it can be seen from table 7 above that compared result among different mode, the production surplus in ACS and ECS just has a slight increase, while in contrast, the Consumer surplus of passengers has a significant increase, which makes the social welfare of the group in ACS and ECS mode much higher than that in the CTS mode. It also demonstrates from the mathematical point of view why the application of mobile travel has been popularized rapidly in recent years. The essence is to enhance the residual value of both the supply and demand sides and create a win-win strategy.

Table 6 The key parameters of Shenzhen city taxi market

\begin{tabular}{cccccc}
\hline Parameters & $\omega$ & $\xi$ & $\alpha$ & $\beta$ & $\gamma$ \\
\hline \multirow{4}{*}{ Parameters } & $\mathbf{4 5 0 6 0}$ & $\mathbf{1 5 8 6}$ & $-\mathbf{1 . 3}$ & $\mathbf{- 0 . 2}$ & -1 \\
& $\kappa$ & $\lambda$ & $c^{1}$ & $c^{2}$ & $c^{3}$ \\
Parameters & $\mathbf{5}$ & $\mathbf{0 . 0 1}$ & $\mathbf{4 . 0}$ & $\mathbf{3 . 0}$ & $\mathbf{5 . 0}$ \\
& $f^{1}$ & $f^{2}$ & $f^{3}$ & & \\
& $\mathbf{7 . 0}$ & $\mathbf{6 . 2}$ & $\mathbf{2 . 0}$ & & \\
\hline
\end{tabular}

Table 7 The social welfare situation (the case of Shenzhen)

$\begin{array}{cccc}\text { Surplus } & \text { Production Surplus } S^{p} & \text { Consumer Surplus } S^{c} & \text { Producer-consumer Surplus } S \\ \text { CTS } & 7840 & 38569 & 46409 \\ \text { ACS } & 9561 & 164352 & 173913 \\ \text { ECS } & 8100 & 159001 & 167101\end{array}$

\subsection{Network and Key Parameters}

In order to show the impact of the mobility loading rate on social welfare more intuitively, we obtain the change of mobility market surplus in different modes, which can be shown in figure 3, 4,5 respectively.As shown in Fig.3, the mobility service surplus is negative in different service mode when the loading rate is low, but it increases first with the increase of the real load rate, and then achieve a maximum value in loading rate is $70 \%$ $80 \%$ of CTS, ACS and ERS. Which indicate the optimal loading rate for mobility service. Otherwise, It can be seen from figure 4 that consumer surplus of passengers decreases continuously with the increase of loading rate in different mode and the range tends to be stable. This is because the decrease of vacant mileages will lead to the longer waiting time and less mobility demand. It also can be shown that the lowest benefit loading rate in CTS, ACS and ETS is $29.2 \%, 42.5$ and $54.2 \%$ from Fig. 3 when mobility service surplus is zero..For the group social welfare in the Fig.5. when the loading rate of CTS mode is very low(under $10 \%$ ), the social welfare CTS mode will increase, but this state is unstable and rarely exists in practice (at this time, the CTS mode is unprofitable) The total social welfare in ACS and ETS is higher than that of the CTS mode no matter what the loading rate is, which proves the role of information and self- driving technology in promoting the social welfare of users. 


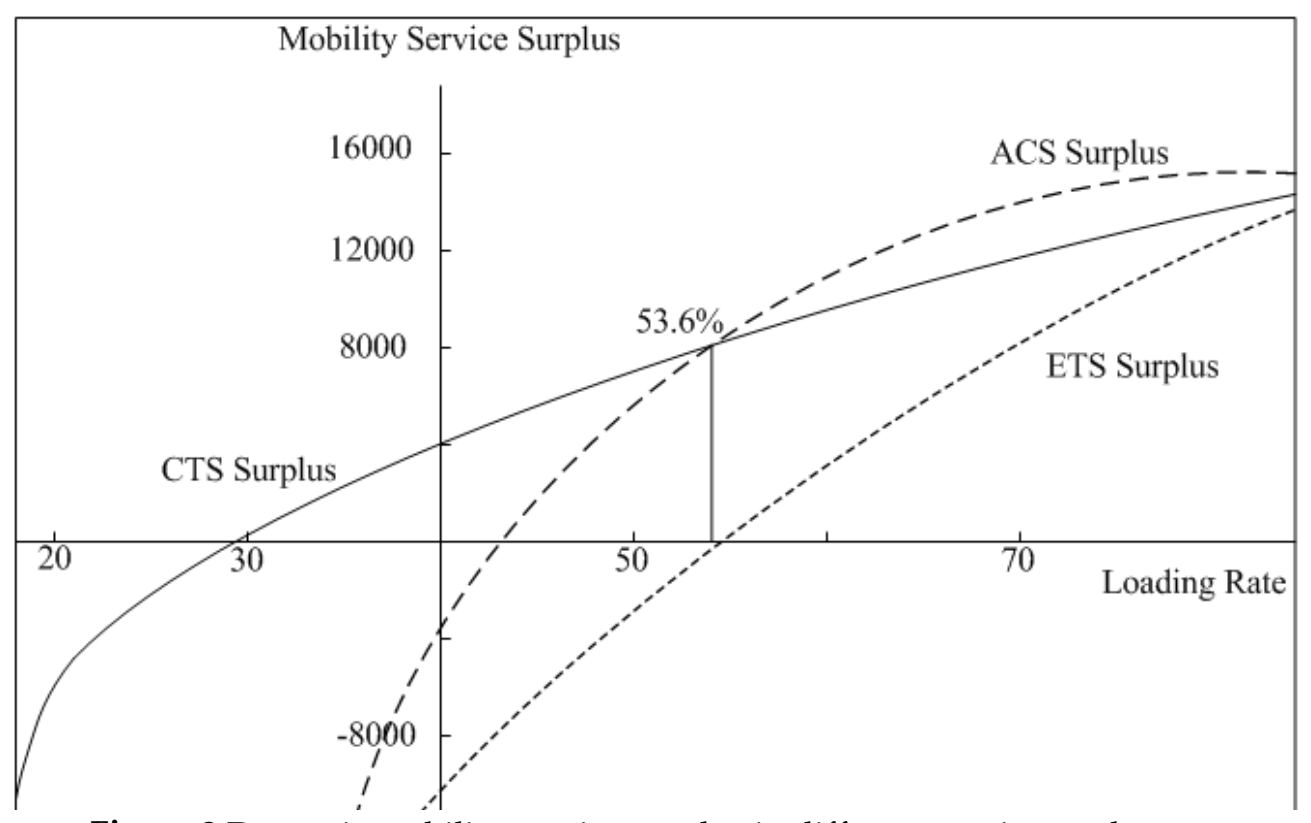

Figure 3 Dynamic mobility service surplus in different service mode

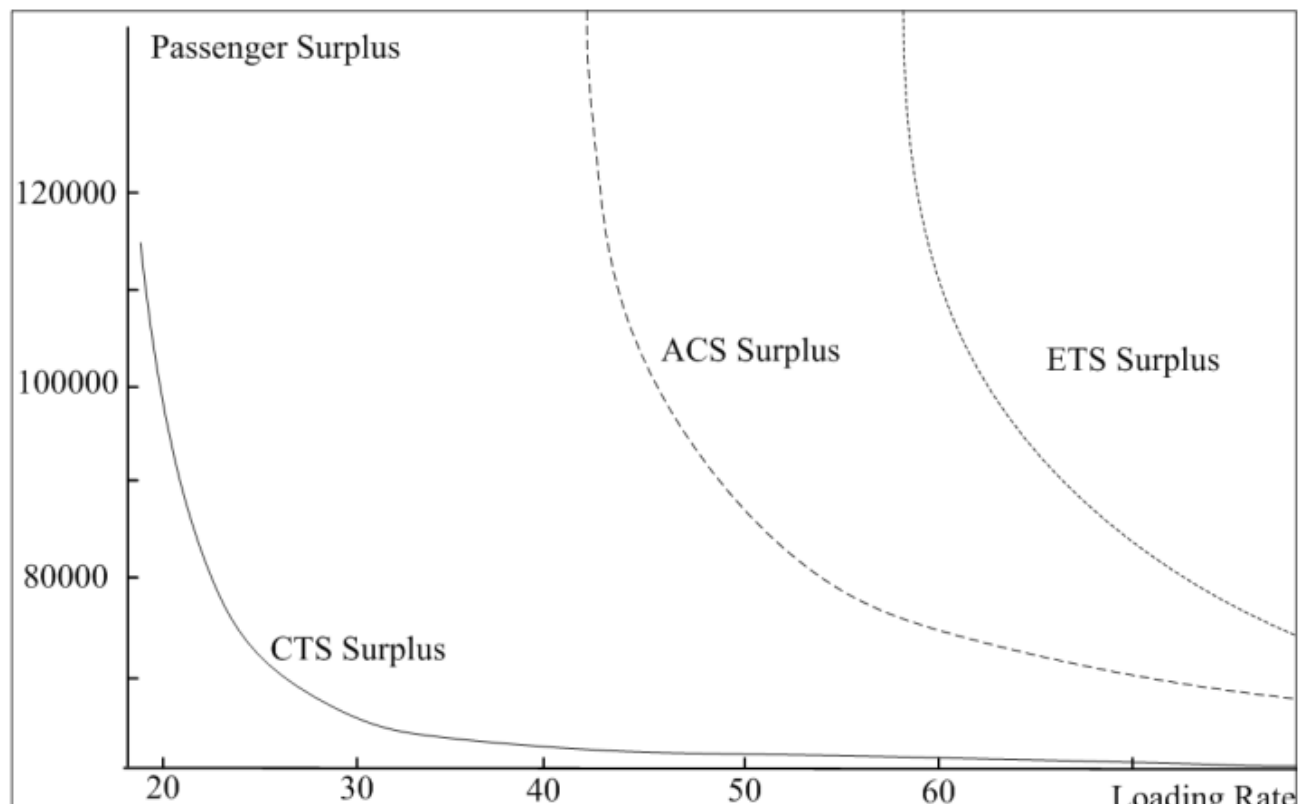

Figure 4 Dynamic passenger surplus in different service mode 


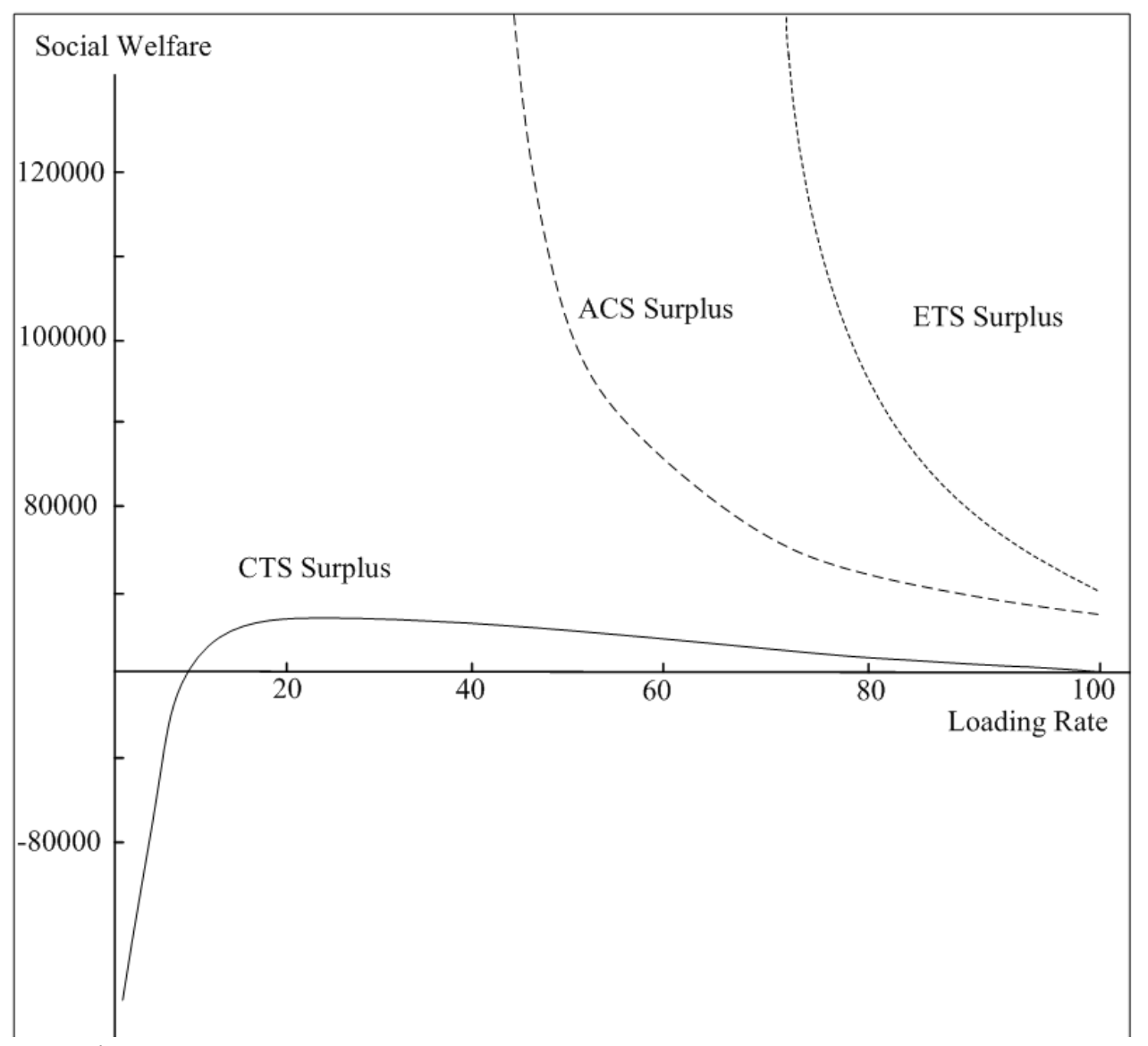

Figure 5 Dynamic social welfare in different service mode

\section{Conclusion}

With growing congestion and seemingly constant disruption in our cities and on our roads and public transport, car-based mobility service is a way to address the lack of capacity in our infrastructure network. As population growth places mounting pressure on CTS mode, ACS and ETS is a new technical advancement to improve the experience for everyone who relies on the MOD for city's transport infrastructure.This paper identified the vacant trip and loading rate evaluation model under mobility oriented development mode. recommended solutions to enhance the effective operation of the different mobility services under numerical illustrations. The total social welfare in ACS and ETS is higher than that of the CTS mode no matter what the loading rate is, which proves the role of information symmetry and self- driving technology in promoting the social welfare of users. By the way it will be our future research considering the congestion situation and passengers' utilities influencing factors.

\section{References}

1. Douglas, G.W. 1972. "Price regulation and optimal service standards : the taxi cab industry." Journal of transport economics and policy 6 (2):116-117.

2. Djavadian, S., and J. Y. J. Chow. 2017. "An agent-based day-to-day adjustment process for modeling 'Mobility as a Service' with a two-sided flexible transport market." Transportation Research Part B: Methodological 104:36-57. 'dio': 10.1016/j.trb.2017.06.015.

3. Erhardt, G. D., S. Roy, D. Cooper, B. Sana, M. Chen, and J. Castiglione. 2019. "Do transportation network companies decrease or increase congestion?" Science Advances 5 (5). 'dio': 10.1126/sciadv.aau2670. 
4. Ma, Jie, Min Xu, Qiang Meng, and Lin Cheng. 2020. "Ridesharing user equilibrium problem under OD-based surge pricing strategy." Transportation Research Part B: Methodological 134:1-24. 'dio': https://doi.org/10.1016/j.trb.2020.02.001.

5. Ramezani, M., and M. Nourinejad. 2018. "Dynamic modeling and control of taxi services in large-scale urban networks: A macroscopic approach." Transportation Research Part C: Emerging Technologies 94:203-219. 'dio': 10.1016/j.trc.2017.08.011.

6. Wang, D., W. Cao, J. Li, and J. Ye. 2017. "DeepSD: Supply-demand prediction for online carhailing services using deep neural networks." Proceedings - International Conference on Data Engineering, 2017-01-01.

7. Wang, Jing-Peng, Xuegang Jeff Ban, and Hai-Jun Huang. 2019. "Dynamic ridesharing with variable-ratio charging-compensation scheme for morning commute." Transportation Research Part B: Methodological 122:390-415. 'dio': https://doi.org/10.1016/j.trb.2019.03.006.

8. Wang, X., W. Liu, H. Yang, D. Wang, and J. Ye. 2020. "Customer behavioural modelling of order cancellation in coupled ride-sourcing and taxi markets." Transportation Research Part B: Methodological 132:358-378. 'dio': 10.1016/j.trb.2019.05.016.

9. Wong, k.1., Wong, S.C and Yang, H., 2001. "Modeling urban taxi services in congested road networks with elastic demand." Transportation Research Part B: Methodological 35 (9):819-842. 'dio': 10.1016/S0191-2615(00)00021-7.

10. Xu, Min, and Qiang Meng. 2019. "Fleet sizing for one-way electric carsharing services considering dynamic vehicle relocation and nonlinear charging profile." Transportation Research Part B: Methodological 128:23-49. 'dio': https://doi.org/10.1016/j.trb.2019.07.016.

11. Yang, H., M. Ye, W. H. Tang, and S. C. Wong. 2005. "Regulating taxi services in the presence of congestion externality." Transportation Research Part A: Policy and Practice 39 (1):17-40. 'dio': 10.1016/j.tra.2004.05.004.

12. Zhou, T., W. Shi, X. Liu, F. Tao, Z. Qian, and R. Zhang. 2019. "A Novel Approach for Online Car-Hailing Monitoring Using Spatiotemporal Big Data." IEEE Access 7:128936-128947. 'dio': 10.1109/ACCESS.2019.2939787. 